\title{
Features of Host Cell Invasion by Different Infective Forms of Trypanosoma cruzi
}

\author{
Renato A Mortara ${ }^{+}$, Daniela O Procópio, Helena C Barros, Newton V \\ Verbisck, Walter K Andreoli, Ricardo BS Silva, Solange da Silva
}

Disciplina de Parasitologia, Escola Paulista de Medicina, Universidade Federal de São Paulo, Rua Botucatu 862, $6^{\circ}$ andar, 04023-062 São Paulo SP Brasil

Through its life cycle from the insect vector to mammalian hosts Trypanosoma cruzi has developed clever strategies to reach the intracellular milieu where it grows sheltered from the hosts' immune system. We have been interested in several aspects of in vitro interactions of different infective forms of the parasite with cultured mammalian cells. We have observed that not only the classically infective trypomastigotes but also amastigotes, originated from the extracellular differentiation of trypomastigotes, can infect cultured cells. Interestingly, the process of invasion of different parasite infective forms is remarkably distinct and also highly dependent on the host cell type.

Key words: Trypanosoma cruzi - cell invasion - amastigote - trypomastigote

In the natural course of infection, the infected insect transmits metacyclic trypomastigotes that invade mammalian cells, escape the endocytic vacuole and transform into rounded amastigote forms. Amastigotes replicate in the cytoplasm, differentiate back into trypomastigotes that are released after cell lysis. An alternative sub-cycle can occur in the mammalian host if amastigotes, originated from premature lysis of infected cells (Behbehani 1973, Nogueira \& Cohn 1976, Carvalho et al. 1981, Umezawa et al. 1985, Ulisses de Carvalho \& De Souza 1986) or by extracellular differentiation of trypomastigotes (Pan 1978, Andrews et al. 1987, Ley et al. 1988, Mortara 1991), invade professional or non-professional phagocytes. Inside these cells the amastigotes can survive and sustain the parasite's life cycle. These amastigote-like infective forms present morphological, immunological, biochemical and ultrastructural similarities to intracellular amastigotes (Villalta \& Kierszenbaum, 1982, 1984, Hudson et al. 1984, Andrews et al. 1987, Ley et al. 1988) and express specific molecular markers of amastigotes (Andrews et al. 1987, Lima et al. 1988, Pan \& McMahon-Pratt 1989). We have seen that extracellular amastigotes are able to infect $\mathrm{HeLa}$ cells by interacting with microvilli on the dorsal surface of these cells (Mortara 1991). This association leads to microvillus aggregation that can be followed by microfila-

\footnotetext{
${ }^{+}$Corresponding author. Fax: +55-11-571-1095. E-mail: renato.dmip@epm.br

Received 9 June 1999

Accepted 9 August 1999
}

ment clustering observed by phalloidin staining. By contrast, trypomastigotes preferentially enter HeLa cells at the edges showing that the different forms of the parasite interact with specific regions of the cell surface (Mortara 1991). Differences in invasion mechanisms utilized by extracellular amastigotes and metacyclic trypomastigotes became even more apparent when we tested the requirement for integrity of host cells' cytoskeleton. For that HeLa and Vero cells were treated with drugs that disrupt microfilaments or microtubules. Mammalian cells with altered expression in actinbinding proteins (Actin-Binding Protein, ABP, and gelsolin) were also tested as target cells. Protein kinase inhibitors (staurosporine and genistein) interfered with the invasion processes. The distribution of different host cell components during the invasion by the two parasite forms was consistent with the emerging notion that extracellular amastigotes and metacyclic trypomastigotes utilize mechanisms to invade host cells with highly distinctive features for each Trypanosoma cruzi form and for each host cell. Preliminary data from our studies also suggest that culture-derived trypomastigotes and metacyclic trypomastigotes may form parasito-phorous vacuoles with distinct properties.

\section{MATERIALS AND METHODS}

Vero, HeLa, Actin-binding mutants and transfectants, gelsolin transfectants, Coxiella burnetti-chronically infected Vero cells were routinely grown in DME or RPMI-1640 media supplemented with fetal calf serum and antibiotics when necessary. Parasites from different $T$. cruzi strains were maintained in LIT medium and trypo- 
mastigotes generated by infecting Vero cells. Amastigotes were obtained from infected cells (intracellular) or after $48 \mathrm{hr}$ incubation of tissue-culture trypomastigotes in LIT medium at $37^{\circ} \mathrm{C}$ (extracellular).

\section{RESULTS AND DISCUSSION}

We have started our study trying to understand how extracellular amastigotes invade mammalian cells. Earlier work from several groups indicated that this is a common observation and we decided to initiate our studies by comparing the mechanism of cell invasion with that of metacyclic trypomastigotes, the vectorial infective form extensively studied by our colleagues.

It was initially observed that intracellular amastigotes of several strains were poorly infective to non-phagocytic cells and extracellular amastigotes of the highly infective CL strain were much less invasive than G-strain derived parasites. Interestingly, the infectivity to HeLa cells of extracellular amastigotes correlated with the expression of a carbohydrate epitope defined by monoclonal antibody 1D9 (Barros et al. 1997, Verbisck et al. 1998) (Figure).

The infectivity of extracellular amastigotes and metacyclic trypomastigotes of the $\mathrm{G}$ strain towards different mammalian target cells was then systematically examined. We observed that amastigotes normally displayed a higher capability of invasion on most cells. The effect of drugs that interfere with microfilament (cytochalasin D) or microtu-

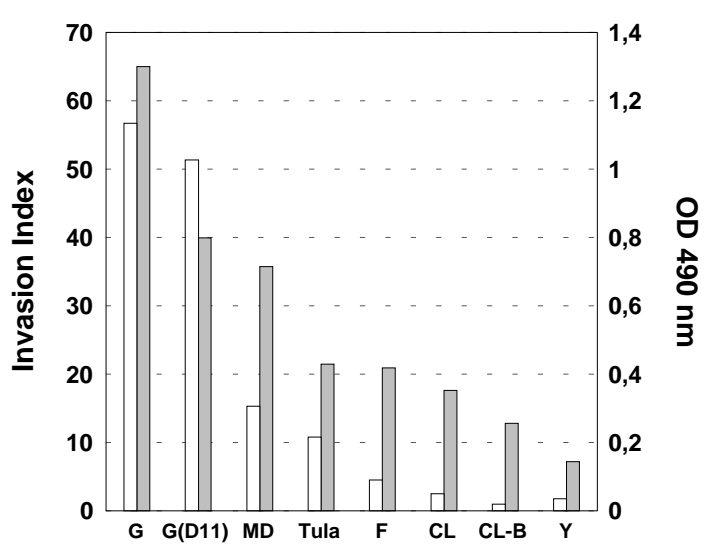

Expression of epitopes defined by Mab 1D9 and infectivity to HeLa cells of extracellular amastigotes of different Trypanosoma cruzi strains and clones. The expression of the epitopes of the indicated MAbs was examined in amastigotes of different strains and clones (expressed as OD $490 \mathrm{~nm}$, grey bars). Extracellular amastigotes were tested for in vitro invasion of HeLa cells and the intracellular parasites scored in at least 400 cells, determined in at least three independent experiments. Invasion index calculated as in (Procópio et al. 1998) are expressed as open bars. bule (nocodazole) assembly on the invasion of the two infective forms was also not only cell (HeLa or Vero) but also infective form dependent (Procópio et al. 1998).

We also tested cells with modified expression in microfilament-associated components (gelsolin and actin-binding protein, ABP) as target cells and again observed contrasting results with the two infective forms of the parasite. Drugs that interfere with protein phosphorylation (genistein and staurosporine) also displayed contrasting effects (Procópio et al. 1998).

The recruitment of extracellular matrix components of HeLa and Vero cells, their integrin receptors and cytoskeletal elements was examined under electron and confocal fluorescence microscopy and significant differences were again found in the pattern involving either trypomastigote or extracellular amastigote invasion (Procópio et al. 1998).

We have also began to examine the formation and escape from the parasitophorous vacuole by the infective forms. We observed that whilst extracellular amastigotes and metacyclic trypomastigotes are found in LAMP-1 positive compartments after $1 \mathrm{hr}$ following infection, extracellular amastigotes appear to escape faster from the vacuole than metecyclics do. Moreover, preliminary results using Vero cells chronically infected with the ryckettsia $C$. burnetti that induce the formation of large and highly fusogenic cytoplasmic vacuoles (Veras et al. 1994, 1995) has disclosed an unforeseen feature of trypomastigote invasion. Indeed, metacyclic trypomastigotes (CL strain) are much more efficiently transferred from the extracellular medium to the $C$. burnetti vacuole than the corresponding tissue-culture derived trypomastigotes under identical conditions. If this observation is confirmed, it would imply that the composition of the parasitophorous membrane involving the two types of trypomastigotes is distinct.

These studies have thus emphasized the notion that the mechanism involved in the entry of different $T$. cruzi infective forms depends not only on the host cell but also on the parasite stage.

\section{REFERENCES}

Andrews NW, Hong K, Robbins ES, Nussenzweig V 1987. Stage-specific surface antigens expressed during the morphogenesis of vertebrate forms of Trypanosoma cruzi. Exp Parasitol 64: 474-484.

Barros HC, Verbisck NV, Silva S, Araguth MF, Mortara RA 1997. Distribution of epitopes of Trypanosoma cruzi amastigotes during the intracellular life cycle within mammalian cells. $J$ Euk Microbiol 46: 332344. 
Behbehani K 1973. Developmental cycles of Trypanosoma (Schyzotrypanum) cruzi (Chagas 1909) in mouse peritoneal macrophages in vitro. Parasitology 66: 343-353.

Carvalho RMG, Meirelles MNL, De Souza W, Leon W 1981. Isolation of the intracellular stage of Trypanosoma cruzi and its interaction with mouse macrophages in vitro. Infect Immun 33: 546-554.

Hudson L, Snary D, Morgan SJ 1984. Trypanosoma cruzi: continuous cultivation with murine cell lines. Parasitology 88: 283-294.

Ley V, Andrews NW, Robbins ES, Nussenzweig V 1988. Amastigotes of Trypanosoma cruzi sustain an infective cycle in mammalian cells. J Exp Med 168: 649659.

Lima MF, Beltz LA, Kierszenbaum F 1988. Trypanosoma cruzi: a specific surface marker for the amastigote form. J Protozool 35: 108-110.

Mortara RA 1991. Trypanosoma cruzi: amastigotes and trypomastigotes interact with different structures on the surface of HeLa cells. Exp Parasitol 73: 1-14.

Nogueira N, Cohn Z 1976. Trypanosoma cruzi: mechanism of entry and intracellular fate in mammalian cells. J Exp Med 143: 1402-1420.

Pan AA, McMahon-Pratt D 1989. Amastigote and epimastigote stage-specific components of Trypanosoma cruzi characterized by using monoclonal antibodies. J Immunol 143: 1001-1008.

Pan SCT 1978. Trypanosoma cruzi: In vitro interactions between cultured amastigotes and human skinmuscle cells. Exp Parasitol 45: 274-286.

Procópio DO, Silva S, Cunningham CC, Mortara RA 1998. Trypanosoma cruzi: Effect of protein kinase inhibitors and cytoskeletal protein organization and expression on host cell invasion by amastigotes and metacyclic trypomastigotes. Exp Parasitol 90: 1-13.

Ulisses de Carvalho TM, De Souza W 1986. Infectivity of amastigotes of Trypanosoma cruzi. Rev Inst Med Trop São Paulo 28: 205-212.

Umezawa ES, Milder RV, Abrahamsohn IA 1985. Trypanosoma cruzi amastigotes: development in vitro and infectivity in vivo of the forms isolated from spleen and liver. Acta Trop 42: 25-32.

Veras PST, De Chastellier C, Moreau, MF, Villiers V, Thibon M, Mattei DM, Rabinovitch M 1994. Fusion between large phagocytic vesicles: Targeting of yeast and other particulates to phagolysosomes that shelter the bacterium Coxiella burnetii or the protozoan Leishmania amazonensis in Chinese hamster ovary cells. J Cell Sci 107: 3065-3076.

Veras PST, Moulia C, Dauguet C, Tunis CT, Thibon M, Rabinovitch M 1995. Entry and survival of Leishmania amazonensis amastigotes within phagolysosome-like vacuoles that shelter Coxiella burnetii in Chinese hamster ovary cells. Infect Immun 63: 35023506.

Verbisck NV, Silva S, Mortara RA 1998. Trypanosoma cruzi: amastigote polymorphism defined by monoclonal antibodies. Braz J Med Biol Res 31: 15831591.

Villalta F, Kierszenbaum F 1982. Growth of isolated amastigotes of Trypanosoma cruzi in cell-free medium. J Protozool 29: 570-576.

Villalta F, Kierszenbaum F 1984. Role of inflammatory cells in Chagas' disease. II. Interactions of mouse macrophages and human monocytes with intracellular forms of Trypanosoma cruzi: uptake and mechanism of destruction. J Immunol 133: 3338-3343. 